\title{
About some basic concepts for metrology in analytical chemistry
}

\author{
Paul De Bièvre
}

Published online: 11 May 2012

(C) Springer-Verlag 2012

There should be renewed attention for 'counting' as form of measurement [1] to serve, for example, in microbiology where counting of bacteria is important, or in studying chemical reactions between protein molecules where counting 'active sites' on the surfaces of these molecules is revealed to be a simple way to measure the potential for interacting of these molecules in the human body. In such cases, expressing measurement results in terms of the quantity 'mass' and its units - be it $\mathrm{kg}$ or $\mathrm{mg}$ - or of the quantity 'amount-of-substance' and its units-be it mole or mmol, is not very helpful. Doing that in terms of 'number of entities' (entry 1.4 Note 3 in [2]) with unit 'one' (symbol ' 1 ') (entry 1.10 Note 3 in [2]) is more appropriate and ... simpler. Thus, it is good to remind ourselves of the 'particulate' or 'granular' structure of matter, an inherent property very different from the property of 'mass'. It entails the usefulness and desirability of-indeed-counting of things: atoms, molecules, ions, bacteria, active sites, but also phenomena such as events, all of them being covered by the generic term 'entities'. Entities are nothing new: we have been measuring radioactive decay in terms of number of events (in becquerel), or in terms of number of decay products (such as number of daughter nuclides). We are thinking in terms of a number of periods of a specified radiation in the ${ }^{133} \mathrm{Cs}$ atom, or express 'indications' (entry 4.1 in [2]) in counts per second in digital outputs, all of them covered by the term 'entities'.

Disclaimer: The author is a member of the Joint Committee on Guides for Metrology (JCGM), Working Group 2 (VIM). The opinions expressed in this Column do not necessarily represent the view of the Working Group nor of ACQUAL.

P. De Bièvre $(\square)$

Kasterlee, Belgium

e-mail: paul.de.bievre@skynet.be
On the macroscopic scale, 'counting' must surely have been the earliest - and the easiest-form of measurement: counting cattle, soldiers, horses, apples, or coconuts, which determined degree of richness or power. A more modern application of counting occurs in banks daily and on a massive scale: counting currency units.

[Note that we always have to specify the name of the entities concerned.]

Thus, 'granularity' is a property of matter [3], naturally leading to coining the concept of a 'number of things'.

That was ultimately recognized in analytical chemistry. For centuries, matter was looked upon as something being 'continuous'. Although Demokritos in ancient Hellas implied already the 'granular' nature of matter, it took Dalton, Avogadro, Cannizzaro, Berzelius, Lavoisier and other chemists, about two thousand years later, to definitively establish that matter had a 'granular' structure. That was even before the physicists coined the very concept of the modern 'atom'. Hence, it was logical-and simple-to arrive at an appropriate unit for counting a number of particles (generic name: entities): 'one'. Granularity being recognized as a phenomenon in nature, the concept 'one entity' to describe the molecular world, appears difficult when we have to study and describe the interaction of very large numbers of molecules. However, that can be easily remedied by defining a fixed multiple of the natural unit 'one' and establish that as a convenient unit for handling and describing these interactions on the macroscopic level. For convenience, we can choose to give that number a name: in the mind of an analytical chemist, that is a 'mole' (symbol: 'mol'). We can replace counting a very large number by measuring ratios of very large numbers. It reduces the problem of measuring very large ratios to measuring number-ratios. An example of that is what we do in a titration. The 'equivalence point' tells us when a 
large but unknown number of ions is equivalent, in fact equal, to a large but known number of other ions, yielding the simplest number-ratio of all: $1 / 1$ (i.e. ' 1 '). In isotope dilution mass spectrometry IDMS, we do something similar: we compare a large but unknown number of atoms of one isotope of an element (in the "unknown sample to be measured") to a known number of atoms of another isotope of the same element (called a 'spike', present in what is usually called a spike solution). Also, here, we have an "equivalence point" or indicator of equality of large numbers: a number-ratio $1 / 1$ (i.e. ' 1 '). Present isotope mass spectrometers are 'measuring systems' (entry 2.3 in [2]) in which the indications (entry 4.1 in [2]) (of ion currents) are linear over a large range and that enables to measure ratios of large numbers (of the isotopic atoms involved) differing from that number-ratio 1 by several orders of magnitude, for example, 1/99 or 999/1, which can be measured easily and very precisely (even very accurately if calibrated) because the quantities being measured have now become electric currents and their ratios.

Thinking in 'number of entities' brings a very clarifying insight to the modern analytical chemist: it does not need the concept of 'amount-of-substance': we did not need it in the above text. In addition, 'amount-of-substance' has sometimes been described as 'the quantity of which the mole is the unit'. That is a reasoning which requires to choose the unit (the mole) and define it before the related concept ('amount-of-substance') can be described. The logical here should be that we first choose and describe the quantity we have in mind, before we assign a unit to it.

The introduction of intercontinentally agreed definitions of concepts such as 'quantity', 'base quantity', 'base unit' in VIM [2] is of great help in clarifying our thinking and in our description of chemical phenomena as well as in expressing measurement results in analytical chemistry.

Comments and opinions set forward here are very much encouraged and can be sent, as usual, to the author or to the Editors.

\section{References}

1. De Bièvre P (2006) Counting is measuring. Accred Qual Assur $11: 1-2$

2. BIPM, IEC, IFCC, ILAC, IUPAC, IUPAP, ISO, OIML (2012) The international vocabulary of metrology-basic and general concepts and associated terms (VIM), 3rd edn. JCGM 200:2012. http://www. bipm.org/vim accessed on 2012-04-15

3. De Bièvre P (2007) Numerosity versus mass. Accred Qual Assur $12: 221-222$ 